J. Korean Math. Soc. 49 (2012), No. 5, pp. 977-991

http://dx.doi.org/10.4134/JKMS.2012.49.5.977

\title{
ON TRANSVERSALLY HARMONIC MAPS OF FOLIATED RIEMANNIAN MANIFOLDS
}

\author{
Min Joo Jung and Seoung Dal Jung
}

\begin{abstract}
Let $(M, \mathcal{F})$ and $\left(M^{\prime}, \mathcal{F}^{\prime}\right)$ be two foliated Riemannian manifolds with $M$ compact. If the transversal Ricci curvature of $\mathcal{F}$ is nonnegative and the transversal sectional curvature of $\mathcal{F}^{\prime}$ is nonpositive, then any transversally harmonic map $\phi:(M, \mathcal{F}) \rightarrow\left(M^{\prime}, \mathcal{F}^{\prime}\right)$ is transversally totally geodesic. In addition, if the transversal Ricci curvature is positive at some point, then $\phi$ is transversally constant.
\end{abstract}

\section{Introduction}

Transversally harmonic maps of foliated Riemannian manifolds were introduced by Konderak and Wolak [6] in 2003. Let $(M, \mathcal{F})$ and $\left(M^{\prime}, \mathcal{F}^{\prime}\right)$ be two foliated Riemannian manifolds and let $\phi: M \rightarrow M^{\prime}$ be a smooth foliated map, i.e., $\phi$ is a smooth leaf-preserving map. Then $\phi$ is said to be transversally harmonic if the transversal tension field $\tau_{b}(\phi)$ vanishes. See Section 3 and [6] for details. Equivalently, it is a critical point of the transversal energy functional on any compact domain of $M$, which is defined in Section 4 (cf. [7]). Also, transversally harmonic maps are considered as harmonic maps between the leaf spaces $[6,7]$. So, for the point foliation, transversally harmonic maps are harmonic maps. Therefore transversally harmonic maps are considered as generalizations of harmonic maps. In this paper, we study transversally harmonic maps and give some interesting facts relating to them. The paper is organized as follows. In Section 2, we review the well-known facts on a foliated Riemannian manifold. In Section 3, we review the properties of the transversally harmonic map, which were studied in [7] and give some results. In Section 4 , we give a new proof of the first normal variational formula for the transversal energy $E_{B}(\phi)$ (Theorem 4.1). In the last section, we study the generalized Weitzenböck formula and give some applications (Theorem 5.3 and Theorem $5.4)$.

Received January 20, 2011; Revised January 31, 2012.

2010 Mathematics Subject Classification. 53C12, 58E20.

Key words and phrases. transversal tension field, transversally harmonic map, normal variational formula, generalized Weitzenböck type formula.

(C)2012 The Korean Mathematical Society 


\section{Preliminaries}

Let $(M, g, \mathcal{F})$ be a $(p+q)$-dimensional foliated Riemannian manifold with a foliation $\mathcal{F}$ of codimension $q$ and a bundle-like metric $g$ with respect to $\mathcal{F}$ $[8,13]$. A foliated Riemannian manifold means a Riemannian manifold with a Riemannian foliation. Let $T M$ be the tangent bundle of $M, L$ the tangent bundle of $\mathcal{F}$, and $Q=T M / L$ the corresponding normal bundle of $\mathcal{F}$. Then we have an exact sequence of vector bundles

$$
0 \longrightarrow L \longrightarrow T M \underset{\sigma}{\stackrel{\pi}{\rightleftarrows}} Q \longrightarrow 0,
$$

where $\pi: T M \rightarrow Q$ is a projection and $\sigma: Q \rightarrow L^{\perp}$ is a bundle map satisfying $\pi \circ \sigma=i d$. Let $g_{Q}$ be the holonomy invariant metric on $Q$ induced by $g=$ $g_{L}+g_{L^{\perp}} ;$ that is,

$$
g_{Q}(s, t)=g(\sigma(s), \sigma(t)) \quad \forall s, t \in \Gamma Q .
$$

This means that $\theta(X) g_{Q}=0$ for $X \in \Gamma L$, where $\theta(X)$ is the transverse Lie derivative. So we have an identification $L^{\perp}$ with $Q$ via an isometric splitting $\left(Q, g_{Q}\right) \cong\left(L^{\perp}, g_{L^{\perp}}\right)$. We denote by $\nabla^{Q}$ the transverse Levi-Civita connection on the normal bundle $Q[13,14]$. The transversal curvature tensor $R^{Q}$ of $\nabla^{Q} \equiv \nabla$ is defined by $R^{Q}(X, Y)=\left[\nabla_{X}, \nabla_{Y}\right]-\nabla_{[X, Y]}$ for any $X, Y \in \Gamma T M$. It is trivial that $i(X) R^{Q}=0$ for any $X \in \Gamma L$, where $i(X)$ is the interior product. Let $K^{Q}, \operatorname{Ric}^{Q}$ and $\sigma^{Q}$ be the transversal sectional curvature, transversal Ricci operator and transversal scalar curvature with respect to $\nabla$, respectively. The foliation $\mathcal{F}$ is said to be minimal if $\kappa=0$, where $\kappa$ is the mean curvature form of $\mathcal{F}$ [13]. Let $\Omega_{B}^{r}(\mathcal{F})$ be the space of all basic $r$-forms, i.e., $\phi \in \Omega_{B}^{r}(\mathcal{F})$ if and only if $i(X) \phi=0$ and $\theta(X) \phi=0$ for any $X \in \Gamma L$. Then $\Omega^{*}(M)=\Omega_{B}^{*}(\mathcal{F}) \oplus \Omega_{B}^{*}(\mathcal{F})^{\perp}$ [1]. Let $\kappa_{B}$ be the basic part of $\kappa$. Then $\kappa_{B}$ is closed, i.e., $d \kappa_{B}=0$ [1]. Now, we define the basic Laplacian $\Delta_{B}$ acting on $\Omega_{B}^{*}(\mathcal{F})$ by

$$
\Delta_{B}=d_{B} \delta_{B}+\delta_{B} d_{B}
$$

where $\delta_{B}$ is the formal adjoint of $d_{B}=\left.d\right|_{\Omega_{B}^{*}(\mathcal{F})}[11]$. Let $\left\{E_{a}\right\}_{a=1, \ldots, q}$ be a local orthonormal basic frame on $Q$. We define $\nabla_{\mathrm{tr}}^{*} \nabla_{\mathrm{tr}}: \Omega_{B}^{r}(\mathcal{F}) \rightarrow \Omega_{B}^{r}(\mathcal{F})$ by

$$
\nabla_{\mathrm{tr}}^{*} \nabla_{\mathrm{tr}}=-\sum_{a} \nabla_{E_{a}, E_{a}}^{2}+\nabla_{\kappa_{B}^{\sharp}}
$$

where $\nabla_{X, Y}^{2}=\nabla_{X} \nabla_{Y}-\nabla_{\nabla_{X}^{M} Y}$ for any $X, Y \in \Gamma T M$ and $\nabla^{M}$ denotes the Levi-Civita connection of $M$. Then the operator $\nabla_{\mathrm{tr}}^{*} \nabla_{\mathrm{tr}}$ is positive definite and formally self adjoint on the space of basic forms [3]. Let $V(\mathcal{F})$ be the space of all transversal infinitesimal automorphisms $Y$ of $\mathcal{F}$, i.e., $[Y, Z] \in \Gamma L$ for all $Z \in \Gamma L[5]$. Let

$$
\bar{V}(\mathcal{F})=\{\bar{Y}=\pi(Y) \mid Y \in V(\mathcal{F})\} .
$$

Note that $\bar{V}(\mathcal{F}) \cong \Omega_{B}^{1}(\mathcal{F})[10]$. For later use, we recall the transversal divergence theorem [16] on a foliated Riemannian manifold. 
Theorem 2.1 (Transversal divergence theorem). Let $\left(M, g_{M}, \mathcal{F}\right)$ be a closed, oriented Riemannian manifold with a transversally oriented foliation $\mathcal{F}$ and $a$ bundle-like metric $g_{M}$ with respect to $\mathcal{F}$. Then

$$
\int_{M} \operatorname{div}_{\nabla} \bar{X}=\int_{M} g_{Q}\left(\bar{X}, \kappa_{B}^{\sharp}\right)
$$

for all $X \in V(\mathcal{F})$, where $\operatorname{div}_{\nabla} X$ denotes the transversal divergence of $X$ with respect to the connection $\nabla$.

Now we define the bundle map $A_{Y}: \Lambda^{r} Q^{*} \rightarrow \Lambda^{r} Q^{*}$ for any $Y \in V(\mathcal{F})[5]$ by

$$
A_{Y} \phi=\theta(Y) \phi-\nabla_{Y} \phi .
$$

It is well-known [5] that for any $s \in \Gamma Q$

$$
A_{Y} s=-\nabla_{Y_{s}} \bar{Y}
$$

where $Y_{s}$ is the vector field such that $\pi\left(Y_{s}\right)=s$. So $A_{Y}$ depends only on $\bar{Y}=\pi(Y)$. Since $\theta(X) \phi=\nabla_{X} \phi$ for any $X \in \Gamma L, A_{Y}$ preserves the basic forms and depends only on $\bar{Y}$. Now, we recall the generalized Weitzenböck formula on $\Omega_{B}^{*}(\mathcal{F})$.

Theorem $2.2([3])$. On a foliated Riemannian manifold $(M, \mathcal{F})$, we have

$$
\Delta_{B} \phi=\nabla_{\mathrm{tr}}^{*} \nabla_{\mathrm{tr}} \phi+F(\phi)+A_{\kappa_{B}^{\sharp}} \phi, \quad \phi \in \Omega_{B}^{r}(\mathcal{F}),
$$

where $F(\phi)=\sum_{a, b} \theta^{a} \wedge i\left(E_{b}\right) R^{\nabla}\left(E_{b}, E_{a}\right) \phi$. If $\phi$ is a basic 1-form, then $F(\phi)^{\sharp}=$ $\operatorname{Ric}^{Q}\left(\phi^{\sharp}\right)$.

Now we recall a very important lemma for later use. From Proposition 4.1 in [11], it is well-known that $\Delta_{B}-\kappa_{B}^{\sharp}$ on all basic functions is the restriction of $\Delta-\kappa^{\sharp}$ on all functions. Hence, by maximum and minimum principles, we have the following lemma.

Lemma $2.3([4])$. Let $(M, g, \mathcal{F})$ be a compact Riemannian manifold with a foliation $\mathcal{F}$ and a bundle-like metric $g$. If $\left(\Delta_{B}-\kappa_{B}^{\sharp}\right) f \geq 0($ or $\leq 0)$ for any basic function $f$, then $f$ is constant.

\section{Transversally harmonic maps}

Let $(M, g, \mathcal{F})$ and $\left(M^{\prime}, g^{\prime}, \mathcal{F}^{\prime}\right)$ be two foliated Riemannian manifolds. Let $\nabla^{M}$ and $\nabla^{M^{\prime}}$ be the Levi-Civita connections of $M$ and $M^{\prime}$, respectively. Let $\nabla$ and $\nabla^{\prime}$ be the transverse Levi-Civita connections on $Q$ and $Q^{\prime}$, respectively. Let $\phi:(M, g, \mathcal{F}) \rightarrow\left(M^{\prime}, g^{\prime}, \mathcal{F}^{\prime}\right)$ be a smooth foliated map, i.e., $d \phi(L) \subset L^{\prime}$. Then we define $d_{T} \phi: Q \rightarrow Q^{\prime}$ by

$$
d_{T} \phi:=\pi^{\prime} \circ d \phi \circ \sigma .
$$

Then $d_{T} \phi$ is a section in $Q^{*} \otimes \phi^{-1} Q^{\prime}$, where $\phi^{-1} Q^{\prime}$ is the pull-back bundle on $M$. Let $\nabla^{\phi}$ and $\tilde{\nabla}$ be the connections on $\phi^{-1} Q^{\prime}$ and $Q^{*} \otimes \phi^{-1} Q^{\prime}$, respectively. Then 
a foliated map $\phi:(M, \mathcal{F}) \rightarrow\left(M^{\prime}, \mathcal{F}^{\prime}\right)$ is called transversally totally geodesic if it satisfies

$$
\tilde{\nabla}_{\operatorname{tr}} d_{T} \phi=0
$$

where $\left(\tilde{\nabla}_{\operatorname{tr}} d_{T} \phi\right)(X, Y)=\left(\tilde{\nabla}_{X} d_{T} \phi\right)(Y)$ for any $X, Y \in \Gamma Q$. Note that if $\phi$ : $M \rightarrow M^{\prime}$ is transversally totally geodesic with $d \phi(Q) \subset Q^{\prime}$, then, for any transversal geodesic $\gamma$ in $M, \phi \circ \gamma$ is also transversal geodesic. The transversal tension field of $\phi$ is defined by

$$
\tau_{b}(\phi)=\operatorname{tr}_{Q} \tilde{\nabla} d_{T} \phi=\sum_{a=1}^{q}\left(\tilde{\nabla}_{E_{a}} d_{T} \phi\right)\left(E_{a}\right),
$$

where $\left\{E_{a}\right\}$ is a local orthonormal basic frame of $Q$. Trivially, the transversal tension field $\tau_{b}(\phi)$ is a section of $\phi^{-1} Q^{\prime}$.

Definition 3.1. Let $\phi:(M, g, \mathcal{F}) \rightarrow\left(M^{\prime}, g^{\prime}, \mathcal{F}^{\prime}\right)$ be a smooth foliated map. Then $\phi$ is said to be transversally harmonic if the transversal tension field of $\phi$ vanishes, i.e., $\tau_{b}(\phi)=0$.

Now we recall the O'Neill tensors $\mathcal{A}$ and $\mathcal{T}[9]$ on a foliated manifold $(M, \mathcal{F})$, which are defined by

$$
\begin{aligned}
& \mathcal{A}_{X} Y=\pi^{\perp}\left(\nabla_{\pi(X)}^{M} \pi(Y)\right)+\pi\left(\nabla_{\pi(X)}^{M} \pi^{\perp}(Y)\right), \\
& \mathcal{T}_{X} Y=\pi^{\perp}\left(\nabla_{\pi^{\perp}(X)}^{M} \pi(Y)\right)+\pi\left(\nabla_{\pi^{\perp}(X)}^{M} \pi^{\perp}(Y)\right)
\end{aligned}
$$

for any $X, Y \in \Gamma T M$, where $\pi^{\perp}: T M \rightarrow L$. It is well-known [9] that

$$
\mathcal{A}_{\pi(X)} \pi(Y)=\pi^{\perp}[\pi(X), \pi(Y)]
$$

for any vector fields $X, Y$ on $M$. Then $\mathcal{T} \equiv 0$ is equivalent to the property that all leaves of $\mathcal{F}$ are totally geodesic submanifolds of $(M, g)$ and $\mathcal{A} \equiv 0$ is equivalent to the integrability of $Q$.

Let $\left\{E_{i}\right\}_{i=1, \ldots, p}$ be a local orthonormal basis of $L$ and $\left\{E_{a}\right\}_{a=1, \ldots, q}$ be a local orthonormal basic frame on $Q$. Then we have the following.

Theorem 3.2. Let $\phi:(M, g, \mathcal{F}) \rightarrow\left(M^{\prime}, g^{\prime}, \mathcal{F}^{\prime}\right)$ be a smooth foliated map. Then

$$
\begin{aligned}
\tau(\phi)= & \tau\left(\left.\phi\right|_{\mathcal{F}}\right)+\tau_{b}(\phi)-d_{T} \phi\left(\kappa^{\sharp}\right)+\operatorname{tr}_{g} \phi^{*} \mathcal{T}^{\prime}+\operatorname{tr}_{Q} \phi^{*} \mathcal{A}^{\prime} \\
& +\sum_{a}\left\{\pi^{\perp} \nabla_{\pi d \phi\left(E_{a}\right)}^{M^{\prime}} \pi^{\perp} d \phi\left(E_{a}\right)+\pi^{\perp} \nabla_{\pi^{\perp} d \phi\left(E_{a}\right)}^{M^{\prime}} \pi^{\perp} d \phi\left(E_{a}\right)-\pi^{\perp} d \phi\left(\nabla_{E_{a}} E_{a}\right)\right\} \\
& +\sum_{a} \pi \nabla_{\pi^{\perp} d \phi\left(E_{a}\right)}^{M^{\prime}} \pi d \phi\left(E_{a}\right),
\end{aligned}
$$

where $\tau(\phi)$ is the tension field of $\phi$ and

$$
\tau\left(\left.\phi\right|_{\mathcal{F}}\right)=\pi^{\perp} \sum_{i}\left(\tilde{\nabla}_{E_{i}} d \phi\right)\left(E_{i}\right) .
$$


Proof. Let $\left\{E_{i}, E_{a}\right\}_{i=1, \ldots, p ; a=1, \ldots, q}$ be a local orthonormal frame of $T M$ such that $E_{i} \in \Gamma L, E_{a} \in \Gamma Q$. By the definition of the tension field, we have

$$
\tau(\phi)=\sum_{i=1}^{p}\left(\tilde{\nabla}_{E_{i}} d \phi\right)\left(E_{i}\right)+\sum_{a=1}^{q}\left(\tilde{\nabla}_{E_{a}} d \phi\right)\left(E_{a}\right) .
$$

Since $\phi$ is a foliated map, $\pi d \phi\left(E_{i}\right)=0$ and $\pi^{\perp} d \phi\left(E_{i}\right)=d \phi\left(E_{i}\right)$. Therefore, we have

$$
\sum_{i=1}^{p}\left(\tilde{\nabla}_{E_{i}} d \phi\right)\left(E_{i}\right)=\tau\left(\left.\phi\right|_{\mathcal{F}}\right)+\sum_{i}\left\{\pi \nabla_{d \phi\left(E_{i}\right)}^{M^{\prime}} d \phi\left(E_{i}\right)-\pi d \phi\left(\nabla_{E_{i}}^{M} E_{i}\right)\right\}
$$

and

$$
\begin{aligned}
& \sum_{a=1}^{q}\left(\tilde{\nabla}_{E_{a}} d \phi\right)\left(E_{a}\right) \\
= & \tau_{b}(\phi)+\sum_{a}\left\{\pi^{\perp} \nabla_{\pi d \phi\left(E_{a}\right)}^{M^{\prime}} \pi d \phi\left(E_{a}\right)+\nabla_{\pi d \phi\left(E_{a}\right)}^{M^{\prime}} \pi^{\perp} d \phi\left(E_{a}\right)\right\} \\
& +\sum_{a}\left\{\nabla_{\pi^{\perp} d \phi\left(E_{a}\right)}^{M^{\prime}} \pi d \phi\left(E_{a}\right)+\nabla_{\pi^{\perp} d \phi\left(E_{a}\right)}^{M^{\prime}} \pi^{\perp} d \phi\left(E_{a}\right)-\pi^{\perp} d \phi\left(\nabla_{E_{a}}^{M} E_{a}\right)\right\} .
\end{aligned}
$$

From (3.6), we have $\pi^{\perp} \nabla_{\pi d \phi\left(E_{a}\right)}^{M^{\prime}} \pi d \phi\left(E_{a}\right)=\pi^{\perp} \nabla_{E_{a}}^{M} E_{a}=0$. Hence, from (3.4) and (3.5), we have

$$
\begin{aligned}
\tau(\phi)= & \tau\left(\left.\phi\right|_{\mathcal{F}}\right)+\tau_{b}(\phi)-\pi d \phi\left(\sum_{i} \pi\left(\nabla_{E_{i}}^{M} E_{i}\right)\right)+\sum_{i} \mathcal{T}_{d \phi\left(E_{i}\right)}^{\prime} d \phi\left(E_{i}\right) \\
& +\sum_{a}\left\{\mathcal{T}_{d \phi\left(E_{a}\right)} d \phi\left(E_{a}\right)+\mathcal{A}_{d \phi\left(E_{a}\right)}^{\prime} d \phi\left(E_{a}\right)+\pi \nabla_{\pi^{\perp} d \phi\left(E_{a}\right)}^{M^{\prime}} \pi d \phi\left(E_{a}\right)\right\} \\
& +\sum_{a} \pi^{\perp}\left\{\nabla_{\pi^{\perp} d \phi\left(E_{a}\right)}^{M^{\prime}} \pi^{\perp} d \phi\left(E_{a}\right)+\nabla_{\pi d \phi\left(E_{a}\right)}^{M^{\prime}} \pi^{\perp} d \phi\left(E_{a}\right)-d \phi\left(\pi \nabla_{E_{a}}^{M} E_{a}\right)\right\}
\end{aligned}
$$

Since $\sum_{i} \pi\left(\nabla_{E_{i}}^{M} E_{i}\right)=\kappa^{\sharp}$, the proof is completed.

Corollary $3.3([15])$. Let $\phi:(M, g, \mathcal{F}) \rightarrow\left(M^{\prime}, g^{\prime}, \mathcal{F}^{\prime}\right)$ be a smooth foliated map and $d \phi(Q) \subset Q^{\prime}$. Then

$$
\tau(\phi)=\tau\left(\left.\phi\right|_{\mathcal{F}}\right)+\tau_{b}(\phi)-d \phi\left(\kappa^{\sharp}\right)+\operatorname{tr}_{L} \phi^{*} \mathcal{T}^{\prime},
$$

where $\operatorname{tr}_{L} \phi^{*} \mathcal{T}^{\prime}=\sum_{i=1}^{p} \mathcal{T}_{d \phi\left(E_{i}\right)}^{\prime} d \phi\left(E_{i}\right)$.

Proof. Since $d \phi(Q) \subset Q^{\prime}, \pi^{\perp} d \phi\left(E_{a}\right)=0$ for all a. Moreover, from (3.5) and (3.6), $\mathcal{A}^{\prime}{ }_{X} X=0$ and $\mathcal{T}^{\prime}{ }_{X} Y=0$ for any $X, Y \in \Gamma Q^{\prime}$. From Theorem 3.2, the desired result follows.

Corollary 3.4 $([7])$. Let $\phi:(M, g, \mathcal{F}) \rightarrow\left(M^{\prime}, g^{\prime}, \mathcal{F}^{\prime}\right)$ be a smooth foliated map and $d \phi(Q) \subset Q^{\prime}$. Assume that $\mathcal{F}$ is minimal and $\mathcal{F}^{\prime}$ is totally geodesic. Then $\phi$ is harmonic if and only if $\phi$ is transversally harmonic and leaf-wise harmonic, i.e., $\tau\left(\left.\phi\right|_{\mathcal{F}}\right)=0$. 
Proof. Since $\mathcal{F}$ is minimal and $\mathcal{F}^{\prime}$ is totally geodesic, i.e, $\kappa=0$ and $\mathcal{T}^{\prime}=0$, by Corollary 3.3, we have

$$
\tau(\phi)=\tau\left(\left.\phi\right|_{\mathcal{F}}\right)+\tau_{b}(\phi)
$$

which yields the results.

Corollary 3.5. Let $\phi:(M, g, \mathcal{F}) \rightarrow\left(M^{\prime}, g^{\prime}, \mathcal{F}^{\prime}\right)$ be a smooth foliated map and $d \phi(Q) \subset Q^{\prime}$. Then $\phi$ is a transversally harmonic map if and only if

$$
\pi(\tau(\phi))=\operatorname{tr}_{L} \phi^{*} \mathcal{T}^{\prime}-d \phi\left(\kappa^{\sharp}\right) .
$$

Now, let $\mathcal{F}$ be a Riemannian flow defined by a unit vector field $V$ on a Riemannian manifold $\left(M^{n+1}, g\right)$. Then

$$
\kappa^{\sharp}=\pi\left(\nabla_{V}^{M} V\right)=\nabla_{V}^{M} V .
$$

In fact, $\nabla_{V}^{M} V$ is already orthogonal to the leaves since $g\left(\nabla_{V}^{M} V, V\right)=0$. Moreover, it is trivial that $\mathcal{F}$ is totally geodesic if and only if $\mathcal{F}$ is minimal, i.e., $\mathcal{T}=0$ if and only if $\kappa^{\sharp}=0$. Let $\mathcal{F}$ and $\mathcal{F}^{\prime}$ be two Riemannian flows defined by unit vector fields $V$ and $V^{\prime}$ on Riemannian manifolds $(M, g)$ and $\left(M^{\prime}, g^{\prime}\right)$, respectively. Let $\phi:(M, \mathcal{F}) \rightarrow\left(M^{\prime}, \mathcal{F}^{\prime}\right)$ be a smooth foliated map. Then

$$
\tau\left(\left.\phi\right|_{\mathcal{F}}\right)=V(\lambda) V^{\prime}-\pi^{\perp} d \phi\left(\kappa^{\sharp}\right), \quad \lambda=\left(\phi^{*} \omega^{\prime}\right)(V),
$$

where $\omega^{\prime}$ is a dual 1-form of $V^{\prime}$. Hence if $d \phi(Q) \subset Q^{\prime}$, then $\phi$ is leaf-wise harmonic if and only if $\lambda$ is basic, i.e., $V(\lambda)=0$. Hence we have the following corollary.

Corollary 3.6. Let $\mathcal{F}$ and $\mathcal{F}^{\prime}$ be two Riemannian flows defined by a unit vector fields $V$ and $V^{\prime}$ on a Riemannian manifolds $M$ and $M^{\prime}$, respectively. Assume that $\mathcal{F}$ and $\mathcal{F}^{\prime}$ are minimal. Let $\phi:(M, g, \mathcal{F}) \rightarrow\left(M^{\prime}, g^{\prime}, \mathcal{F}^{\prime}\right)$ be a smooth foliated map and $d \phi(Q) \subset Q^{\prime}$. Then $\phi$ is harmonic if and only if $\phi$ is transversally harmonic and $\phi^{*}\left(\omega^{\prime}\right)(V)$ is basic.

Proof. Since $\mathcal{F}$ is minimal, from (3.10)

$$
\tau\left(\left.\phi\right|_{\mathcal{F}}\right)=V(\lambda) V^{\prime}, \quad \lambda=\left(\phi^{*} \omega^{\prime}\right)(V) .
$$

Hence the proof follows from Corollary 3.4.

Let $\phi:(M, \mathcal{F}) \rightarrow\left(M^{\prime}, \mathcal{F}^{\prime}\right)$ and $\psi:\left(M^{\prime}, \mathcal{F}^{\prime}\right) \rightarrow\left(M^{\prime \prime}, \mathcal{F}^{\prime \prime}\right)$ be smooth foliated maps. Then the composition $\psi \circ \phi:(M, \mathcal{F}) \rightarrow\left(M^{\prime \prime}, \mathcal{F}^{\prime \prime}\right)$ is a smooth foliated map. Moreover, we have

$$
d_{T}(\psi \circ \phi)=d_{T} \psi \circ d_{T} \phi .
$$

Hence we have the following proposition.

Proposition 3.7. Let $\phi:(M, \mathcal{F}) \rightarrow\left(M^{\prime}, \mathcal{F}^{\prime}\right)$ and $\psi:\left(M^{\prime}, \mathcal{F}^{\prime}\right) \rightarrow\left(M^{\prime \prime}, \mathcal{F}^{\prime \prime}\right)$ be smooth foliated maps. Then

$$
\tilde{\nabla}_{\mathrm{tr}} d_{T}(\psi \circ \phi)=d_{T} \psi\left(\tilde{\nabla}_{\mathrm{tr}} d_{T} \phi\right)+\phi^{*} \tilde{\nabla}_{\mathrm{tr}} d_{T} \psi
$$


where

$$
\left(\phi^{*} \tilde{\nabla}_{\mathrm{tr}} d_{T} \psi\right)(X, Y)=\left(\tilde{\nabla}_{d_{T} \phi(X)} d_{T} \psi\right)\left(d_{T} \phi(Y)\right)
$$

for any $X, Y \in \Gamma Q$.

Proof. From (3.11), we have that, for any $X, Y \in \Gamma Q$,

$$
\begin{aligned}
\left(\tilde{\nabla}_{\operatorname{tr}} d_{T}(\psi \circ \phi)\right)(X, Y) & =\nabla_{X}^{\psi \circ \phi} d_{T}(\psi \circ \phi)(Y)-d_{T}(\psi \circ \phi)\left(\nabla_{X} Y\right) \\
& =\left(\tilde{\nabla}_{d_{T} \phi(X)} d_{T} \psi\right)\left(d_{T} \phi(Y)\right)+d_{T} \psi\left(\left(\tilde{\nabla}_{X} d_{T} \phi\right)(Y)\right) \\
& =\left(\phi^{*} \tilde{\nabla}_{\operatorname{tr}} d_{T} \psi\right)(X, Y)+d_{T} \psi\left(\tilde{\nabla}_{\operatorname{tr}} d_{T} \phi\right)(X, Y),
\end{aligned}
$$

which proves (3.12).

Corollary 3.8. Let $\phi:(M, \mathcal{F}) \rightarrow\left(M^{\prime}, \mathcal{F}^{\prime}\right)$ and $\psi:\left(M^{\prime}, \mathcal{F}^{\prime}\right) \rightarrow\left(M^{\prime \prime}, \mathcal{F}^{\prime \prime}\right)$ be smooth foliated maps. Then the transversal tension field of the composition is given by

$$
\tau_{b}(\psi \circ \phi)=d_{T} \psi\left(\tau_{b}(\phi)\right)+\operatorname{tr}_{Q} \phi^{*} \tilde{\nabla}_{\mathrm{tr}} d_{T} \psi
$$

where

$$
\operatorname{tr}_{Q} \phi^{*} \tilde{\nabla}_{\operatorname{tr}} d_{T} \psi=\sum_{a=1}^{q}\left(\tilde{\nabla}_{d_{T} \phi\left(E_{a}\right)} d_{T} \psi\right)\left(d_{T} \phi\left(E_{a}\right)\right) .
$$

Corollary 3.9. Let $\phi:(M, \mathcal{F}) \rightarrow\left(M^{\prime}, \mathcal{F}^{\prime}\right)$ be a transversally harmonic map and let $\psi:\left(M^{\prime}, \mathcal{F}^{\prime}\right) \rightarrow\left(M^{\prime \prime}, \mathcal{F}^{\prime \prime}\right)$ be a transversally totally geodesic map. Then $\psi \circ \phi:(M, \mathcal{F}) \rightarrow\left(M^{\prime \prime}, \mathcal{F}^{\prime \prime}\right)$ is a transversally harmonic map.

\section{The first normal variational formula}

Let $\phi:(M, g, \mathcal{F}) \rightarrow\left(M^{\prime}, g^{\prime}, \mathcal{F}^{\prime}\right)$ be a smooth foliated map. Let $\Omega$ be a compact domain of $M$. Then the transversal energy of $\phi$ on $\Omega \subset M$ is defined by

$$
E_{B}(\phi ; \Omega)=\frac{1}{2} \int_{\Omega}\left|d_{T} \phi\right|^{2} \mu_{M},
$$

where $\left|d_{T} \phi\right|^{2}=\sum_{a} g_{Q^{\prime}}\left(d_{T} \phi\left(E_{a}\right), d_{T} \phi\left(E_{a}\right)\right)$ and $\mu_{M}$ is the volume element of $M$.

Let $V \in \phi^{-1} Q^{\prime}$. Obviously, $V$ may be considered as a vector field on $Q^{\prime}$ along $\phi$. Then there is a 1-parameter family of foliated maps $\phi_{t}$ with $\phi_{0}=\phi$ and $\left.\frac{d \phi_{t}}{d t}\right|_{t=0}=V$. The family $\left\{\phi_{t}\right\}$ is said to be a foliated variation of $\phi$ with the normal variation vector field $V$. Then we have the first normal variational formula(cf. [7]).

Theorem 4.1. (The first normal variational formula) Let $\phi:(M, \mathcal{F}) \rightarrow$ $\left(M^{\prime}, \mathcal{F}^{\prime}\right)$ be a smooth foliated map. Let $\left\{\phi_{t}\right\}$ be a smooth foliated variation of $\phi$ supported in a compact domain $\Omega$. Then

$$
\left.\frac{d}{d t} E_{B}\left(\phi_{t}, \Omega\right)\right|_{t=0}=-\int_{\Omega}\left\langle V, \tau_{b}(\phi)-d_{T} \phi\left(\kappa_{B}^{\sharp}\right)\right\rangle \mu_{M},
$$


where $V=\left.\frac{d \phi_{t}}{d t}\right|_{t=0}$ is the normal variation vector field of $\left\{\phi_{t}\right\}$ and $\langle\cdot, \cdot\rangle$ is the pull-back metric on $\phi^{-1} Q^{\prime}$.

Proof. Let $\Omega$ be a compact domain of $M$ and let $\left\{\phi_{t}\right\}$ be a foliated variation of $\phi$ supported in $\Omega$ with the normal variation vector field $V \in \phi^{-1} Q^{\prime}$. Choose a local orthonormal basic frame $\left\{E_{a}\right\}$ on $Q$ such that $\left(\nabla E_{a}\right)(x)=0$. Define $\Phi: M \times(-\epsilon, \epsilon) \rightarrow M^{\prime}$ by $\Phi(x, t)=\phi_{t}(x)$ and set $E=\Phi^{-1} Q^{\prime}$. Let $\nabla^{\Phi}$ denote the pull-back connection on $E$. Obviously, $d_{T} \Phi\left(E_{a}\right)=d_{T} \phi_{t}\left(E_{a}\right)$ and $d \Phi\left(\frac{\partial}{\partial t}\right)=\frac{d \phi_{t}}{d t}$. Moreover, we have $\nabla_{\frac{\partial}{\partial t}}^{\Phi} \frac{\partial}{\partial t}=\nabla_{\frac{\partial}{\partial t}}^{\Phi} E_{a}=\nabla_{E_{a}}^{\Phi} \frac{\partial}{\partial t}=0$. Hence we have

$$
\begin{aligned}
\frac{d}{d t} E_{B}\left(\phi_{t}, \Omega\right) & =\int_{\Omega} \sum_{a}\left\langle\nabla_{\frac{\partial}{\partial t}}^{\Phi} d_{T} \Phi\left(E_{a}\right), d_{T} \Phi\left(E_{a}\right)\right\rangle \mu_{M} \\
& =\int_{\Omega} \sum_{a}\left\langle\nabla_{E_{a}}^{\Phi} d \Phi\left(\frac{\partial}{\partial t}\right), d_{T} \Phi\left(E_{a}\right)\right\rangle \mu_{M} \\
& =\int_{\Omega} \sum_{a}\left\{E_{a}\left\langle\frac{d \phi_{t}}{d t}, d_{T} \phi_{t}\left(E_{a}\right)\right\rangle-\left\langle\frac{d \phi_{t}}{d t}, \nabla_{E_{a}}^{\phi_{t}} d_{T} \phi_{t}\left(E_{a}\right)\right\rangle\right\} \mu_{M} \\
& =\int_{\Omega} \sum_{a} E_{a}\left\langle\frac{d \phi_{t}}{d t}, d_{T} \phi_{t}\left(E_{a}\right)\right\rangle \mu_{M}-\int_{\Omega}\left\langle\frac{d \phi_{t}}{d t}, \tau_{b}\left(\phi_{t}\right)\right\rangle \mu_{M}
\end{aligned}
$$

Now we define a normal vector field $W_{t}$ by

$$
W_{t}=\sum_{a}\left\langle\frac{d \phi_{t}}{d t}, d_{T} \phi_{t}\left(E_{a}\right)\right\rangle E_{a} .
$$

Then we have

$$
\operatorname{div}_{\nabla} W_{t}=\sum_{a} E_{a}\left\langle\frac{d \phi_{t}}{d t}, d_{T} \phi_{t}\left(E_{a}\right)\right\rangle
$$

By the transversal divergence theorem (Theorem 2.1), we have

$$
\begin{aligned}
\frac{d}{d t} E_{B}\left(\phi_{t}, \Omega\right) & =\int_{\Omega} \operatorname{div}_{\nabla} W_{t} \mu_{M}-\int_{\Omega}\left\langle\frac{d \phi_{t}}{d t}, \tau_{b}\left(\phi_{t}\right)\right\rangle \mu_{M} \\
& =\int_{\Omega}\left\langle W_{t}, \kappa_{B}^{\sharp}\right\rangle \mu_{M}-\int_{\Omega}\left\langle\frac{d \phi_{t}}{d t}, \tau_{b}\left(\phi_{t}\right)\right\rangle \mu_{M} \\
& =-\int_{\Omega}\left\langle\frac{d \phi_{t}}{d t}, \tau_{b}\left(\phi_{t}\right)-d_{T} \phi\left(\kappa_{B}^{\sharp}\right)\right\rangle \mu_{M},
\end{aligned}
$$

which proves (4.2).

Corollary 4.2. Let $\phi:(M, \mathcal{F}) \rightarrow\left(M^{\prime}, \mathcal{F}^{\prime}\right)$ be a smooth foliated map. Assume that $\mathcal{F}$ is minimal. Then $\phi$ is transversally harmonic if and only if $\phi$ is a critical point of the trasnversal energy of $\phi$ supported in a compact domain. 


\section{A generalized Weitzenböck type formula and its applications}

Let $(M, g, \mathcal{F})$ and $\left(M^{\prime}, g^{\prime}, \mathcal{F}^{\prime}\right)$ be two foliated Riemannian manifolds and let $\phi:(M, \mathcal{F}) \rightarrow\left(M^{\prime}, \mathcal{F}^{\prime}\right)$ be a smooth foliated map. Note that $\left|d_{T} \phi\right|^{2} \in \Omega_{B}^{0}(\mathcal{F})$ [6]. Let $\Omega_{B}^{r}(E)=\Omega_{B}^{r}(\mathcal{F}) \otimes E$ be the space of $E$-valued basic $r$-forms, where $E=\phi^{-1} Q^{\prime}$. We define $d_{\nabla}: \Omega_{B}^{r}(E) \rightarrow \Omega_{B}^{r+1}(E)$ by

$$
d_{\nabla}(\omega \otimes s)=(-1)^{r} \omega \wedge \nabla^{\phi} s+d_{B} \omega \otimes s
$$

for any $s \in \Gamma E$ and $\omega \in \Omega_{B}^{r}(\mathcal{F})$. Let $\delta_{\nabla}$ be a formal adjoint of $d_{\nabla}$. Then we have the following.

$$
d_{\nabla}=\sum_{a} \theta^{a} \wedge \tilde{\nabla}_{E_{a}}, \quad \delta_{\nabla}=-\sum_{a} i\left(E_{a}\right) \tilde{\nabla}_{E_{a}}+i\left(\kappa_{B}^{\sharp}\right),
$$

where $i(X)(\omega \otimes s)=i(X) \omega \otimes s$ for any $X \in \Gamma T M$. Then the Laplacian $\Delta$ on $\Omega_{B}^{*}(E)$ is defined by

$$
\Delta=d_{\nabla} \delta_{\nabla}+\delta_{\nabla} d_{\nabla}
$$

Moreover, the operators $A_{X}$ and $\theta(X)$ are extended to $\Omega_{B}^{r}(E)$ as follows:

$$
\begin{aligned}
A_{X}(\omega \otimes s) & =A_{X} \omega \otimes s, \\
\theta(X)(\omega \otimes s) & =\theta(X) \omega \otimes s+\omega \otimes \nabla_{X}^{\phi} s
\end{aligned}
$$

for any $\omega \otimes s \in \Omega_{B}^{r}(E)$ and $X \in \Gamma T M$. Then $\theta(X)=d_{\nabla} i(X)+i(X) d_{\nabla}$ for any $X \in \Gamma T M$. Hence $\Phi \in \Omega_{B}^{*}(E)$ if and only if $i(X) \Phi=0$ and $\theta(X) \Phi=0$ for all $X \in \Gamma L$. Then the generalized Weitzenböck type formula (2.9) is extended to $\Omega_{B}^{*}(E)$ as follows:

$$
\Delta \Phi=\tilde{\nabla}_{\mathrm{tr}}^{*} \tilde{\nabla}_{\mathrm{tr}} \Phi+F(\Phi)+A_{\kappa_{B}^{\sharp}} \Phi, \quad \forall \Phi \in \Omega_{B}^{r}(E),
$$

where $F(\Phi)=\sum_{a, b=1}^{q} \theta^{a} \wedge i\left(E_{b}\right) \tilde{R}\left(E_{b}, E_{a}\right) \Phi$. Note that $d_{T} \phi \in \Omega_{B}^{1}(E)$. Then we have the following.

Theorem 5.1. Let $\phi:(M, g, \mathcal{F}) \rightarrow\left(M^{\prime}, g^{\prime}, \mathcal{F}^{\prime}\right)$ be a smooth foliated map. Then the generalized Weitzenböck type formula is given by

$$
\frac{1}{2} \Delta_{B}\left|d_{T} \phi\right|^{2}=\left\langle\Delta d_{T} \phi, d_{T} \phi\right\rangle-\left|\tilde{\nabla}_{\mathrm{tr}} d_{T} \phi\right|^{2}-\left\langle A_{\kappa_{B}^{\sharp}} d_{T} \phi, d_{T} \phi\right\rangle-\left\langle F\left(d_{T} \phi\right), d_{T} \phi\right\rangle,
$$

where

$$
\begin{aligned}
& \left\langle F\left(d_{T} \phi\right), d_{T} \phi\right\rangle \\
= & \sum_{a} g_{Q^{\prime}}\left(d_{T} \phi\left(\operatorname{Ric}^{\mathrm{Q}}\left(E_{a}\right)\right), d_{T} \phi\left(E_{a}\right)\right) \\
& -\sum_{a, b} g_{Q^{\prime}}\left(R^{Q^{\prime}}\left(d_{T} \phi\left(E_{b}\right), d_{T} \phi\left(E_{a}\right)\right) d_{T} \phi\left(E_{a}\right), d_{T} \phi\left(E_{b}\right)\right) .
\end{aligned}
$$


Proof. Let $\left\{E_{a}\right\}(a=1, \ldots, q)$ be a local orthonormal basic frame such that at $x \in M,\left(\nabla E_{a}\right)_{x}=0$. Then, at $x$, we have from $(2.4)$

$$
\frac{1}{2} \Delta_{B}\left|d_{T} \phi\right|^{2}=\left\langle\tilde{\nabla}_{\mathrm{tr}}^{*} \tilde{\nabla}_{\mathrm{tr}} d_{T} \phi, d_{T} \phi\right\rangle-\left|\tilde{\nabla}_{\mathrm{tr}} d_{T} \phi\right|^{2} .
$$

From (5.6) and (5.8), we have

$$
\frac{1}{2} \Delta_{B}\left|d_{T} \phi\right|^{2}=\left\langle\Delta d_{T} \phi, d_{T} \phi\right\rangle-\left|\tilde{\nabla}_{\operatorname{tr}} d_{T} \phi\right|^{2}-\left\langle A_{\kappa_{B}^{\sharp}} d_{T} \phi, d_{T} \phi\right\rangle-\left\langle F\left(d_{T} \phi\right), d_{T} \phi\right\rangle .
$$

Now, we compute $\left\langle F\left(d_{T} \phi\right), d_{T} \phi\right\rangle$. Let $\left\{V_{\alpha}\right\}\left(\alpha=1, \ldots, q^{\prime}\right)$ be a local orthonormal basic frame of $Q^{\prime}$ and $\omega^{\alpha}$ be its dual coframe field. Let $f^{\alpha}=\phi^{*} \omega^{\alpha}$. Then $d_{T} \phi$ is expressed by

$$
d_{T} \phi=\sum_{\alpha=1}^{q^{\prime}} f^{\alpha} \otimes V_{\alpha},
$$

where $V_{\alpha}(x) \equiv V_{\alpha}(\phi(x))$. By a direct calculation, we have

$$
\tilde{R}\left(E_{a}, E_{b}\right) d_{T} \phi=\sum_{\alpha} R^{Q}\left(E_{a}, E_{b}\right) f^{\alpha} \otimes V_{\alpha}+\sum_{\alpha} f^{\alpha} \otimes R^{E}\left(E_{a}, E_{b}\right) V_{\alpha},
$$

where $R^{E}\left(E_{a}, E_{b}\right) V_{\alpha}=R^{Q^{\prime}}\left(d_{T} \phi\left(E_{a}\right), d_{T} \phi\left(E_{b}\right)\right) V_{\alpha}$. From (5.10), we have

$$
\begin{aligned}
\left\langle F\left(d_{T} \phi\right), d_{T} \phi\right\rangle= & \left\langle\sum_{a, b} \theta^{a} \wedge i\left(E_{b}\right) \tilde{R}\left(E_{b}, E_{a}\right) d_{T} \phi, d_{T} \phi\right\rangle \\
= & \sum_{a, b, \alpha, \beta}\left\langle\theta^{a} \wedge i\left(E_{b}\right) R^{Q}\left(E_{b}, E_{a}\right) f^{\alpha} \otimes V_{\alpha}, f^{\beta} \otimes V_{\beta}\right\rangle \\
& +\sum_{a, b, \alpha, \beta} g_{Q}\left(\theta^{a} \wedge i\left(E_{b}\right) f^{\alpha}, f^{\beta}\right) g_{Q}\left(R^{E}\left(E_{a}, E_{b}\right) V_{\alpha}, V_{\beta}\right) .
\end{aligned}
$$

Note that $d_{T} \phi\left(E_{a}\right)=\sum_{\alpha} f^{\alpha}\left(E_{a}\right) V_{\alpha}$. Then we have

$$
\begin{aligned}
& \sum_{a, b, \alpha} g_{Q}\left(\theta^{a} \wedge i\left(E_{b}\right) R^{Q}\left(E_{b}, E_{a}\right) f^{\alpha}, f^{\alpha}\right) \\
= & \sum_{a} g_{Q^{\prime}}\left(d_{T}\left(\operatorname{Ric}^{Q}\left(E_{a}\right)\right), d_{T} \phi\left(E_{a}\right)\right) .
\end{aligned}
$$

From (5.11), we have

$$
\begin{aligned}
\left\langle F\left(d_{T} \phi\right), d_{T} \phi\right\rangle= & \sum_{a} g_{Q^{\prime}}\left(d_{T} \phi\left(\operatorname{Ric}^{Q}\left(E_{a}\right)\right), d_{T} \phi\left(E_{a}\right)\right) \\
& -\sum_{a, b} g_{Q^{\prime}}\left(R^{Q^{\prime}}\left(d_{T} \phi\left(E_{a}\right), d_{T} \phi\left(E_{b}\right)\right) d_{T} \phi\left(E_{b}\right), d_{T} \phi\left(E_{a}\right)\right),
\end{aligned}
$$

which completes the proof.

Remark. (1) Let $\phi:(M, \mathcal{F}) \rightarrow\left(M^{\prime}, \mathcal{F}^{\prime}\right)$ be a smooth foliated map. Then

$$
d_{\nabla}\left(d_{T} \phi\right)=0, \quad \delta_{\nabla} d_{T} \phi=-\tau_{b}(\phi)+i\left(\kappa_{B}^{\sharp}\right) d_{T} \phi .
$$


(2) If a foliated map $\phi:(M, \mathcal{F}) \rightarrow\left(M^{\prime}, \mathcal{F}^{\prime}\right)$ is transversally harmonic, then

$$
\Delta d_{T} \phi=d_{\nabla} i\left(\kappa_{B}^{\sharp}\right) d_{T} \phi .
$$

Corollary 5.2. Let $\phi:(M, g, \mathcal{F}) \rightarrow\left(M^{\prime}, g^{\prime}, \mathcal{F}^{\prime}\right)$ be a transversally harmonic map. Then

$$
\frac{1}{2} \Delta_{B}\left|d_{T} \phi\right|^{2}=-\left|\tilde{\nabla}_{\operatorname{tr}} d_{T} \phi\right|^{2}-\left\langle F\left(d_{T} \phi\right), d_{T} \phi\right\rangle+\frac{1}{2} \kappa_{B}^{\sharp}\left(\left|d_{T} \phi\right|^{2}\right) .
$$

Proof. Since $d_{\nabla}\left(d_{T} \phi\right)=0$, we have

$$
A_{X} d_{T} \phi=-\tilde{\nabla}_{X} d_{T} \phi+d_{\nabla} i(X) d_{T} \phi, \quad \forall X \in \Gamma Q .
$$

Hence (5.14) follows from (5.13) and (5.15).

As applications of the generalized Weitzenböck formula, we have the following theorems.

Theorem 5.3. Let $(M, g, \mathcal{F})$ be a compact foliated Riemannian manifold of nonnegative transversal Ricci curvature, and let $\left(M^{\prime}, g^{\prime}, \mathcal{F}^{\prime}\right)$ be a foliated Riemannian manifold of nonpositive transversal sectional curvature. If $\phi:(M, \mathcal{F})$ $\rightarrow\left(M^{\prime}, \mathcal{F}^{\prime}\right)$ is transversally harmonic, then $\phi$ is transversally totally geodesic, i.e., $\nabla_{\mathrm{tr}} d_{T} \phi=0$. Furthermore,

(1) If the transversal Ricci curvature $\operatorname{Ric}^{Q}$ of $\mathcal{F}$ is positive somewhere, then $\phi$ is transversally constant, i.e., the induced map between leaf spaces is constant.

(2) If the transversal sectional curvature $K^{Q^{\prime}}$ of $\mathcal{F}^{\prime}$ is negative, then $\phi$ is either transversally constant or $\phi(M)$ is a transversally geodesic closed curve.

Proof. Let $\phi:(M, \mathcal{F}) \rightarrow\left(M^{\prime}, \mathcal{F}^{\prime}\right)$ be a transversally harmonic map. Then, from (5.14), we have

$$
\frac{1}{2}\left(\Delta_{B}-\kappa_{B}^{\sharp}\right)\left|d_{T} \phi\right|^{2}=-\left|\tilde{\nabla}_{\mathrm{tr}} d_{T} \phi\right|^{2}-\left\langle F\left(d_{T} \phi\right), d_{T} \phi\right\rangle .
$$

Since $\operatorname{Ric}^{Q} \geq 0$ and $K^{Q^{\prime}} \leq 0$, from (5.7) we have

$$
\left\langle F\left(d_{T} \phi\right), d_{T} \phi\right\rangle \geq 0 .
$$

Hence $\left(\Delta_{B}-\kappa_{B}^{\sharp}\right)\left|d_{T} \phi\right|^{2} \leq 0$. From Lemma $2.3,\left|d_{T} \phi\right|$ is constant. Hence again, we have from $(5.16)$

$$
\left|\tilde{\nabla}_{\mathrm{tr}} d_{T} \phi\right|^{2}+\left\langle F\left(d_{T} \phi\right), d_{T} \phi\right\rangle=0 \text {. }
$$

Hence $\tilde{\nabla}_{\text {tr }} d_{T} \phi=0$ and by assumptions

$$
\begin{aligned}
& g_{Q^{\prime}}\left(d_{T} \phi\left(\operatorname{Ric}^{Q}\left(E_{a}\right), d_{T} \phi\left(E_{a}\right)\right)=0,\right. \\
& g_{Q^{\prime}}\left(R^{Q^{\prime}}\left(d_{T} \phi\left(E_{a}\right), d_{T} \phi\left(E_{b}\right)\right) d_{T} \phi\left(E_{a}\right), d_{T} \phi\left(E_{b}\right)\right)=0
\end{aligned}
$$


for any indices $a$ and $b$. Therefore $\phi$ is transversally totally geodesic. Moreover, from (5.19), if $\operatorname{Ric}^{Q}$ is positive at some point, then $d_{T} \phi=0$, i.e., $\phi$ is transversally constant, which proves (1). For the proof of (2), if there exists a point $x \in M$ such that at least two vectors in $\left\{d_{T} \phi\left(E_{a}\right)\right\}$ are linearly independent at $\phi(x)$, say, $d_{T} \phi\left(E_{1}\right)$ and $d_{T} \phi\left(E_{2}\right)$, then from the hypothesis,

$$
g_{Q^{\prime}}\left(R^{Q^{\prime}}\left(d_{T} \phi\left(E_{1}\right), d_{T} \phi\left(E_{2}\right)\right) d_{T} \phi\left(E_{2}\right), d_{T} \phi\left(E_{1}\right)\right)<0,
$$

which contradicts (5.20). Hence the rank of $d_{T} \phi<2$, that is, the rank of $d_{T} \phi$ is zero or one everywhere. If the rank of $d_{T} \phi$ is zero, then $\phi$ is transversally constant. If the rank of $d_{T} \phi$ is one, then $\phi(M)$ is closed transversally geodesic.

Next, we extend Theorem 5.3 under the weaker transversal sectional curvature of $\mathcal{F}^{\prime}$. Let $\operatorname{rank}_{T}(\phi)$ be the rank of $d_{T} \phi$.

Theorem 5.4. Let $(M, g, \mathcal{F})$ be a compact foliated Riemannian manifold and let $\left(M^{\prime}, g^{\prime}, \mathcal{F}^{\prime}\right)$ be a foliated Riemannian manifold. Assume that $\operatorname{Ric}^{Q} \geq \lambda$ id. and $K^{Q^{\prime}} \leq \mu$ for any positive constants $\lambda$ and $\mu$. Let $\phi:(M, \mathcal{F}) \rightarrow$ $\left(M^{\prime}, \mathcal{F}^{\prime}\right)$ be a transversally harmonic map with $\max \left\{\operatorname{rank}_{T}(\phi)\right\} \leq C$, where $C \geq 2$ is constant. If $\left|d_{T} \phi\right|^{2} \leq \frac{\lambda C}{\mu(C-1)}$, then $\phi$ is transversally constant or $\phi$ is transversally totally geodesic. In particular, if $\left|d_{T} \phi\right|^{2} \leq \frac{\lambda}{\mu}$, then $\phi$ is transversally constant.

Proof. Let $\left\{E_{a}\right\}$ be a local orthonormal basic frame of $Q$. From (5.7), we have

$$
\begin{aligned}
& \left\langle F\left(d_{T} \phi\right), d_{T} \phi\right\rangle \\
= & \sum_{a} g_{Q^{\prime}}\left(d_{T} \phi\left(\operatorname{Ric}^{Q}\left(E_{a}\right)\right), d_{T} \phi\left(E_{a}\right)\right) \\
& -\sum_{a, b}\left\{\left|d_{T} \phi\left(E_{a}\right)\right|^{2}\left|d_{T} \phi\left(E_{b}\right)\right|^{2}-g_{Q^{\prime}}\left(d_{T} \phi\left(E_{a}\right), d_{T} \phi\left(E_{b}\right)\right)^{2}\right\} K_{a b}^{Q^{\prime}},
\end{aligned}
$$

where

$$
K_{a b}^{Q^{\prime}}=g_{Q^{\prime}}\left(R^{Q^{\prime}}\left(d_{T} \phi\left(E_{a}\right), d_{T} \phi\left(E_{b}\right)\right) d_{T} \phi\left(E_{b}\right), d_{T} \phi\left(E_{a}\right)\right)
$$

is the transversal sectional curvature spanned by $d_{T} \phi\left(E_{a}\right)$ and $d_{T} \phi\left(E_{b}\right)$. Let $\operatorname{rank}_{T}(\phi)=r \leq C$. Now, we choose a local orthonormal basic frame $\left\{E_{a}\right\}$ such that $\left.g_{Q^{\prime}}\left(d_{T}\left(E_{a}\right), d_{T}\left(E_{b}\right)\right)\right|_{x}=\lambda_{a} \delta_{a b}$ and $\lambda_{1} \geq \lambda_{2} \geq \cdots \geq \lambda_{r}>0$. Then, from (5.14) and (5.21), we have

$$
\begin{aligned}
& \frac{1}{2} \Delta_{B}\left|d_{T} \phi\right|^{2} \\
= & -\left|\tilde{\nabla}_{\operatorname{tr}} d_{T} \phi\right|^{2}+\frac{1}{2} \kappa_{B}^{\sharp}\left(\left|d_{T} \phi\right|^{2}\right)-\sum_{a} g_{Q^{\prime}}\left(d_{T} \phi\left(\operatorname{Ric}^{Q}\left(E_{a}\right)\right), d_{T} \phi\left(E_{a}\right)\right) \\
& +\sum_{a, b}\left\{\left|d_{T} \phi\left(E_{a}\right)\right|^{2}\left|d_{T} \phi\left(E_{b}\right)\right|^{2}-g_{Q^{\prime}}\left(d_{T} \phi\left(E_{a}\right), d_{T} \phi\left(E_{b}\right)\right)^{2}\right\} K_{a b}^{Q^{\prime}}
\end{aligned}
$$




$$
\leq-\left|\tilde{\nabla}_{\mathrm{tr}} d_{T} \phi\right|^{2}+\frac{1}{2} \kappa_{B}^{\sharp}\left(\left|d_{T} \phi\right|^{2}\right)-\lambda\left|d_{T} \phi\right|^{2}+\mu\left\{\left|d_{T} \phi\right|^{4}-\sum_{a=1}^{r} \lambda_{a}^{2}\right\} .
$$

Using the Schwarz's inequality, we have

$$
\begin{aligned}
\left|d_{T} \phi\right|^{4} & =\sum_{a, b}^{r} \lambda_{a} \lambda_{b} \leq \frac{1}{2} \sum_{a, b=1}^{r}\left(\lambda_{a}^{2}+\lambda_{b}^{2}\right) \\
& =r \sum_{a=1}^{r} \lambda_{a}^{2} \leq C \sum_{a=1}^{r} \lambda_{a}^{2}
\end{aligned}
$$

From (5.22), we have

$$
\left|d_{T} \phi\right|^{4}-\sum_{a=1}^{r} \lambda_{a}^{2} \leq \frac{C-1}{C}\left|d_{T} \phi\right|^{4}
$$

From (5.23), we have

$$
\begin{aligned}
& \frac{1}{2} \Delta_{B}\left|d_{T} \phi\right|^{2} \\
\leq & -\left|\tilde{\nabla}_{\mathrm{tr}} d_{T} \phi\right|^{2}+\frac{1}{2} \kappa_{B}^{\sharp}\left(\left|d_{T} \phi\right|^{2}\right)-\left|d_{T} \phi\right|^{2}\left\{\lambda-\frac{(C-1) \mu}{C}\left|d_{T} \phi\right|^{2}\right\} \\
\leq & \frac{1}{2} \kappa_{B}^{\sharp}\left(\left|d_{T} \phi\right|^{2}\right) .
\end{aligned}
$$

Hence, from Lemma 2.3, $\left|d_{T} \phi\right|$ is constant and then

$$
\left|\tilde{\nabla}_{\mathrm{tr}} d_{T} \phi\right|^{2}+\left|d_{T} \phi\right|^{2}\left\{\lambda-\frac{(C-1) \mu}{C}\left|d_{T} \phi\right|^{2}\right\}=0 .
$$

Therefore $\tilde{\nabla}_{\operatorname{tr}} d_{T} \phi=0$ and $\left|d_{T} \phi\right|^{2}\left\{\lambda-\frac{(C-1) \mu}{C}\left|d_{T} \phi\right|^{2}\right\}=0$. Hence $\phi$ is transversally totally geodesic. If $d_{T} \phi=0$, then $\phi$ is transversally constant. If $d_{T} \phi \neq 0$, then $\left|d_{T} \phi\right|^{2}=\frac{\lambda C}{\mu(C-1)}$ and $\phi$ is transversally totally geodesic. In particular, if $\left|d_{T} \phi\right|^{2} \leq \frac{\lambda}{\mu}$, then $\phi$ is transversally constant.

Remark. For the point foliation, Theorem 5.3 and Theorem 5.4 are found in [2, page 124] and [12], respectively.

Example. Let $T^{2}$ be the flat 2-torus parametrized by the angles $(u, v)$ with $0 \leq u, v<2 \pi$. Let $\bar{\phi}: T^{2} \rightarrow S^{3}$ be defined by

$$
\bar{\phi}(u, v)=(\cos u, \sin u, \cos v, \sin v) / \sqrt{2},
$$

considered as a point in $\mathbb{R}^{4}$. Then $\bar{\phi}$ is harmonic but not totally geodesic $[2$, page 132]. Now let $(F, h)$ and $\left(F^{\prime}, h^{\prime}\right)$ be Riemannian manifolds. Consider the foliations on $T^{2} \times F$ and $S^{3} \times F^{\prime}$ given by the projections on the first component $\pi_{1}: T^{2} \times F \rightarrow T^{2}, \pi_{2}: S^{3} \times F^{\prime} \rightarrow S^{3}$, respectively. Then the projections $\pi_{i}(i=1,2)$ are Riemannian fibrations, and so the foliations are 
Riemannian. Let $\phi: T^{2} \times F \rightarrow S^{3} \times F^{\prime}$ be a foliated smooth map, which is given by

$$
\phi((u, v), x)=(\bar{\phi}(u, v), f(u, v, x))
$$

for any $x \in F$, where $f: T^{2} \times F \rightarrow F^{\prime}$ is smooth. Then $\phi$ is transversally harmonic because $\bar{\phi}$ is harmonic. But $\phi$ is not totally geodesic because $\bar{\phi}$ is not totally geodesic [6, Theorem 3.1].

Acknowledgements. The authors would like to thank the referee for his or her significant corrections and kind comments. This research was supported by the Basic Science Research Program through the National Research Foundation of Korea(NRF) funded by the Ministry of Education, Science and Technology(2010-0021005).

\section{References}

[1] J. A. Alvarez López, The basic component of the mean curvature of Riemannian foliations, Ann. Global Anal. Geom. 10 (1992), no. 2, 179-194.

[2] J. Eells and J. H. Sampson, Harmonic mappings of Riemannian manifolds, Amer. J. Math. 86 (1964), 106-160.

[3] S. D. Jung, The first eigenvalue of the transversal Dirac operator, J. Geom. Phys. 39 (2001), no. 3, 253-264.

[4] S. D. Jung, K. R. Lee, and K. Richardson, Generalized Obata theorem and its applications on foliations, J. Math. Anal. Appl. 376 (2011), no. 1, 129-135.

[5] F. W. Kamber and Ph. Tondeur, Infinitesimal automorphisms and second variation of the energy for harmonic foliations, Tôhoku Math. J. (2) 34 (1982), no. 4 525-538.

[6] J. Konderak and R. Wolak, Transversally harmonic maps between manifolds with Riemannian foliations, Q. J. Math. 54 (2003), no. 3, 335-354.

[7] _ Some remarks on transversally harmonic maps, Glasg. Math. J. 50 (2008), no. $1,1-16$.

[8] P. Molino, Riemannian Foliations, translated from the French by Grant Cairns, Boston: Birkhäser, 1988.

[9] B. O'Neill, The fundamental equations of a submersion, Michigan Math. J. 13 (1966), $459-469$.

[10] H. K. Pak and J. H. Park, Transversal harmonic transformations for Riemannian foliations, Ann. Global Anal. Geom. 30 (2006), no. 1, 97-105.

[11] E. Park and K. Richardson, The basic Laplacian of a Riemannian foliation, Amer. J. Math. 118 (1996), no. 6, 1249-1275.

[12] H. C. J. Sealey, Harmonic maps of small energy, Bull. London Math. Soc. 13 (1981), no. $5,405-408$

[13] Ph. Tondeur, Foliations on Riemannian Manifolds, New-York, Springer-Verlag, 1988.

[14] , Geometry of Foliations, Basel: Birkhäuser Verlag, 1997.

[15] Y. L. Xin, Geometry of Harmonic Maps, Birkhäuser, Boston, 1996.

[16] S. Yorozu and T. Tanemura, Green's theorem on a foliated Riemannian manifold and its applications, Acta Math. Hungar. 56 (1990), no. 3-4, 239-245.

Min Joo Jung

Department of Mathematics

Jeju National University

JeJU 690-756, KoREA

E-mail address: niver486@jejunu.ac.kr 
SEOUng Dal Jung

Department of Mathematics and Research Institute for Basic Sciences

Jeju National University

JEJU 690-756, KoREA

E-mail address: sdjung@jejunu.ac.kr 